\title{
Technique of FBG fabrication with an arbitrary spectrum
}

\author{
A. Quintela*, J.M. Lázaro, J. Mirapeix, N. Becue, M. Silva, J.M. López-Higuera \\ Photonic Engineering Group-University of Cantabria, \\ Avda. Los Castros s/n C.P. 39005 Santander, Spain
}

\begin{abstract}
An arbitrary FBG spectrum can be obtained as the addition of the contribution of some concatenated subFBGs written without phase jump using the same phase mask. An adaptive filter is used in order to obtain, from the desired spectrum, the parameters of the different subFBGs reproducing the required response.
\end{abstract}

Keywords: FBG Fabrication, arbitrary spectrum

\section{INTRODUCTION}

In the last years, the investigation in the field of fiber Bragg gratings (FBG) has experimented a great development and has also attracted a considerable attention due to their application in optical communications [1], optic filters [1-2], sensors [3], dispersion compensation [3], lasers [3], etc. However, these studies have been focused on gratings with standard spectral responses without trying to achieve arbitrary spectra that give more versatility and can be used in applications like channel equalization.

The current techniques for FBG writing are designed for standard spectra. Zhiyong Zhao et. al. [4] proposed to combine the response of gratings with different inclinations to obtain a desired spectrum. When the FBG is tilted a displacement in the wavelength and a decrease in the reflectivity are produced. This fact considerably limits this technique. In this paper, a modification of the phase mask technique [5-6] is used to write FBGs with an arbitrary spectrum.

An arbitrary spectral response is obtained by means of the combination of several gratings. In this way, a good combination of spectra approaching the desired spectral response is calculated. The necessary calibrations for a subsequent writing of those spectra in the fiber are carried out. The fabrication method is demonstrated by writing a FBG with an arbitrary M-shape spectrum.

\section{FABRICATION METHOD}

The fabrication method lies in the writing of some concatenated subFBGs, forcing that the global response to be that of the desired spectrum. Initially, it is necessary to know the parameters of the different subFBGs to write. To accomplish this step an adaptive filter is used. The adaptive filter needs as input the desired spectrum and a battery of FBGs spectra. This battery is composed by spectra of standard FBGs with different Bragg wavelengths. An example of spectra battery is shown in Fig 1. The adaptive filter provides for each battery spectrum a factor. With these factors and the characteristics of the battery spectra the characteristics and parameters of the subFBGs, necessary to be written in order to obtain the desired spectrum, are obtained.

The writing technique consists of two passes. In the first one, the photosensitive fiber is insolated by the UV radiation across a phase mask. The scan speed is constant for each subFBG. The reflectivity of each subFBG fixes this speed. In the second pass, without the phase mask, each subFBG is situated at the wavelength determinated by the adaptive filter. This is accomplished by changing the core effective refraction index on each subFBG with an UV radiation at a constant scan speed for each subFBG. In Fig 2 a scheme of the fabrication method is shown.

*quintela@unican.es; phone 34942 201495; fax 34942200877

Third European Workshop on Optical Fibre Sensors, Antonello Cutolo, Brian Culshaw, José Miguel López-Higuera, Eds., Proceedings of SPIE Vol. 6619,66192S, (2007) $\cdot 0277-786 X / 07 / \$ 18 \cdot$ doi: 10.1117/12.738544 


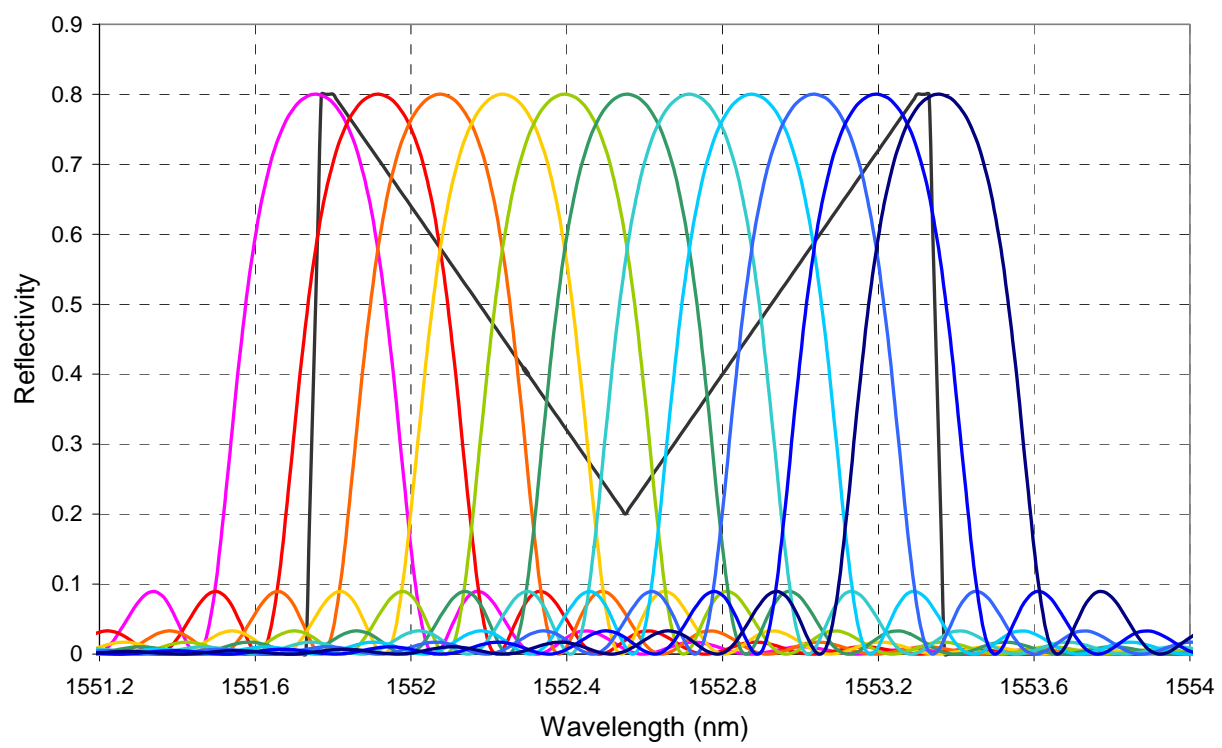

Fig. 1. Example of spectra battery
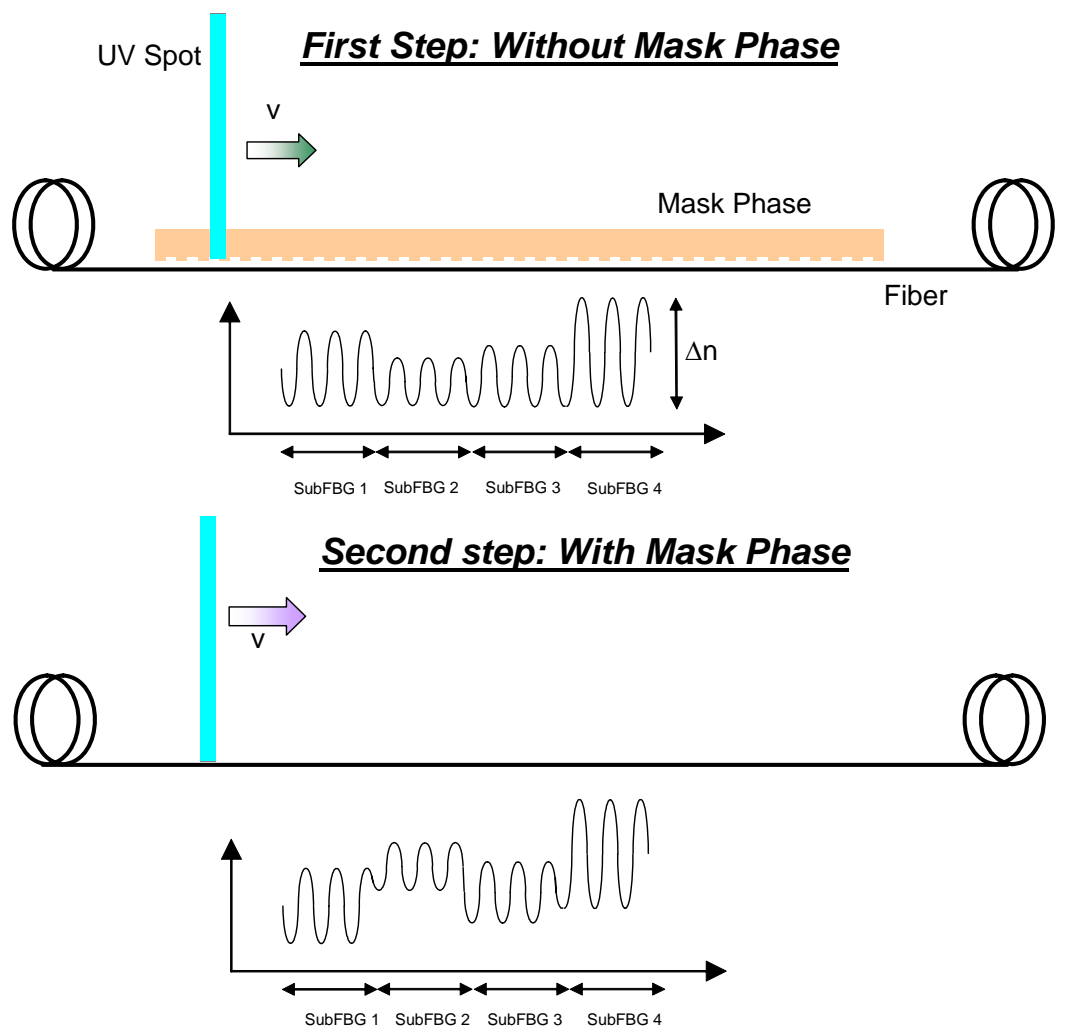

Fig. 2. Scheme of the fabrication method 
In the first pass, after the selection of the appropriated phase mask, it is necessary to obtain the scan speed for each zone. So, from the calculated reflectivities the $\Delta \mathrm{n}$ for each subFBG is obtained, using (1) and (2) [7].

$$
\begin{gathered}
k \cdot L=\tanh ^{-1}\left(\sqrt{R_{\max }}\right) \\
\Delta \mathrm{n}=\frac{1}{\left(\frac{\pi \cdot \mathrm{L}}{\mathrm{k} \cdot \mathrm{L} \cdot \lambda_{\mathrm{B}}}-\frac{1}{n_{e f f}}\right)}
\end{gathered}
$$

From $\Delta \mathrm{n}$ it is possible to calculate the UV exposition time for each point, but a preliminary characterization to know the relation between $\Delta \mathrm{n}$ and the exposition energy for the used installation is needed. The characterization is made in terms of energy in order to be independent of the UV laser power. To obtain this relation some different FBGs with different lengths were written using different UV spot width. In Fig 3a the characterization curve obtained for the first pass is shown.

In the second pass, each subFBG is located at the wavelength obtained from the adaptive filter. To calculate the wavelength shifts it is necessary to know the initial and final wavelength. The initial wavelength depends on the phase mask used and the reflectivity of the subFBG. From the wavelength shift the $\Delta \mathrm{n}$ necessary in each zone is obtained. As in the first step, it is necessary to make a preliminary characterization to obtain the relation between UV exposition energy and $\Delta \mathrm{n}$. The characterization previously made is not valid for the second pass because in the second pass a phase mask is not used, and so the energy in the core of the photosensitive fiber is different. To obtain this relation several standard FBGs, with different lengths, were exposed to UV radiation, with different UV spot width, without a phase mask. The obtained results are shown in Fig $3 \mathrm{~b}$.

a)

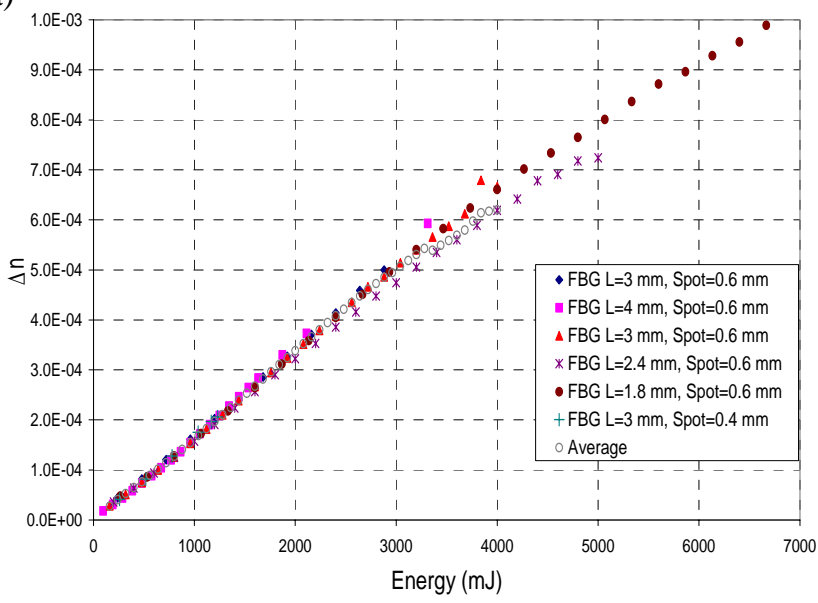

b)

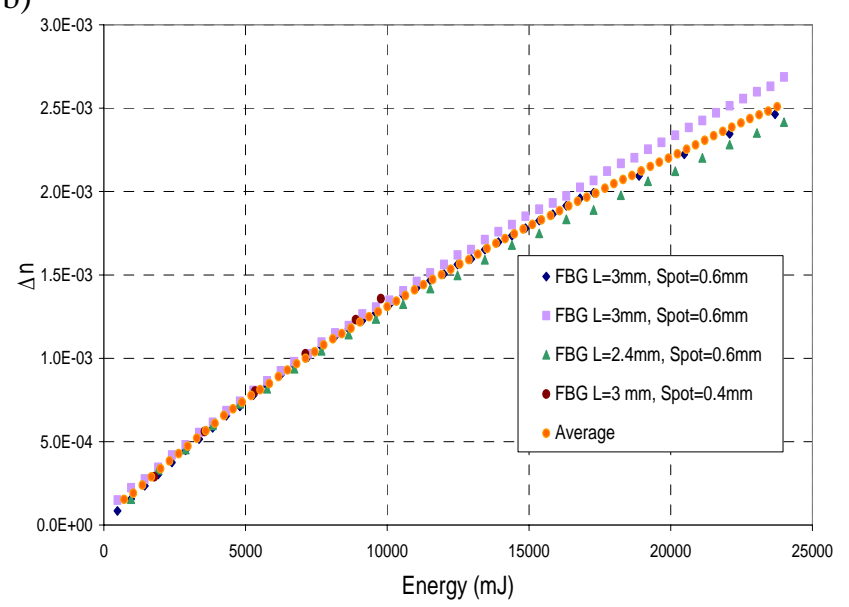

Fig. 3. Characterization graphics: a) $\Delta$ n vs UV exposition energy with phase mask b) $\Delta$ n vs UV exposition energy without phase mask

\section{EXPERIMENTAL}

A FBG with a spectrum with an M-shape was written in order to demonstrate the proposed fabrication method. After some tests it was decided to use a battery of eleven FBGs with a length equal to $3 \mathrm{~mm}$ and $80 \%$ of reflectivity (Fig 1). Using this battery the adaptive filter determined that only five subFBGs, with reflectivities between $75 \%$ and $21 \%$, were necessary to obtain a M-shape spectrum. These five subFBGs were written using the proposed fabrication method. The obtained result is shown in Fig 4, where the M-shape desired spectrum, the simulated spectrum using the responses of the adaptive filter and the spectrum obtained using the proposed fabrication method are presented. It can be observed that there is a good correlation between the measured and the simulated spectra. 


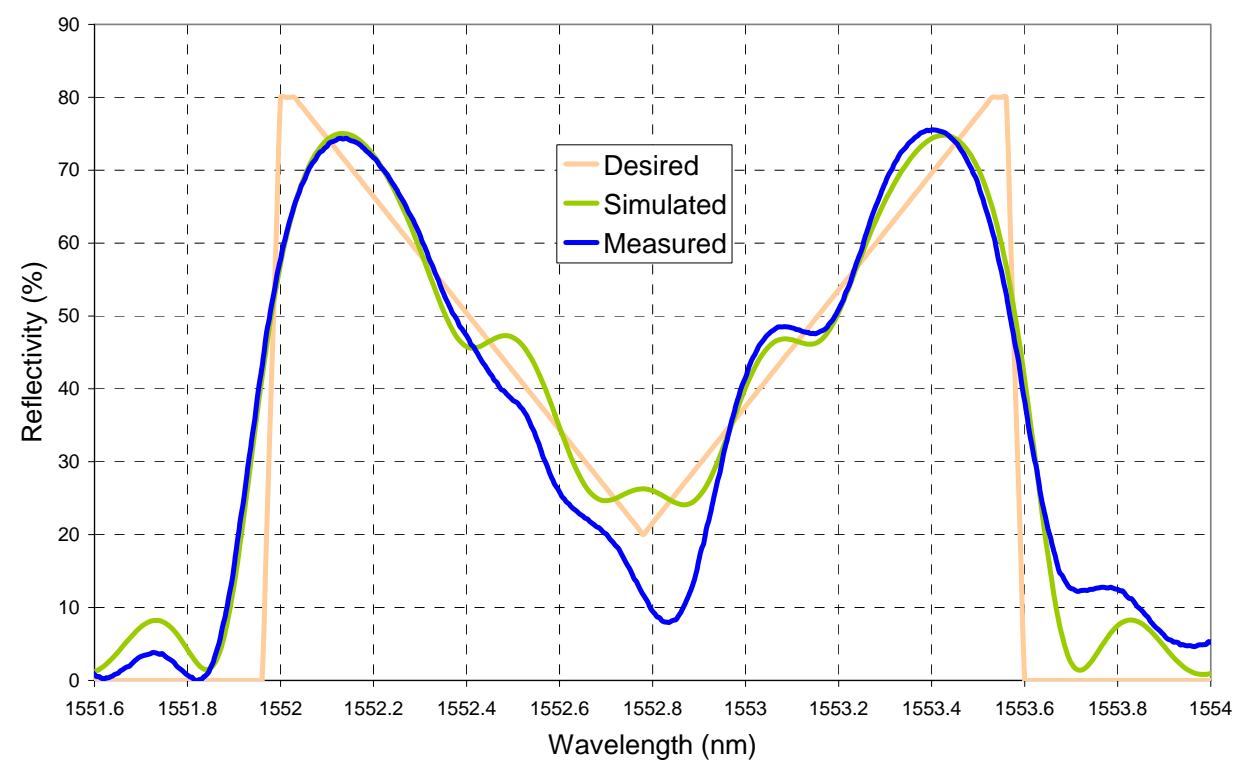

Fig. 4. Desired, simulated and measured spectra.

\section{CONCLUSIONS}

In this paper a FBG fabrication method, allowing to obtain FBGs with an arbitrary spectrum, has been presented and successfully demonstrated. The proposed technique is based on the addition of the contributions of some concatenated subFBGs written without phase shift using only a phase mask. An adaptive filter was used to obtain the characteristics of the concatenated FBGs. The proposed fabrication method has been experimentally demonstrated by writing a FBG successfully reproducing a reflective spectrum modeled as an " $\mathrm{M}$ ".

\section{ACKNOWLEDGMENTS}

This work has been co-supported by the Spanish TEC2004-05936-C02 and TEC2005-08218-C02-02 projects

\section{REFERENCES}

K. O. Hill, D. C. Johnson, F. Bilodeau, and S. Faucher. "Narrow-bandwidth optical waveguide transmission filters: A new design concept and applications to optical fiber communications", Electronics Letters, 23, 465-466, (1987)

Bilodeau, F., et al. "High-Return-Loss narrowband all-fiber bandpass Bragg transmission filter" IEEE Photonics Techn. Letters, 6(1), 80-82, (1994)

J. M. López-Higuera, Editor. Handbook of Optical Fiber sensing Technology, Ed. Wiley \& Sons, 2002.

Zhiyong Zhao, Shuang Zhang, Yongsen Yu, Zhongchang Zhuo, Jian Zhang, Wei Zheng, and Yushu Zhang, "Fabrication of a tilted Bragg grating with a designed reflection spectrum profile" Optics Letters, 29(3), 244-246,(2004)

Hill, K. O., et al. "Brag gratings fabricated in monomode photosensitive optical fiber by UV exposure through a phase mask," Applied Physics Letters, 62, 1035-1037, (1993)

Rourke, H.N., Baker, S.R., Byron, K.C., Baulcomb, R.S., Ojha, S.M., Clements, S., "Fabrication and characterisation of long, narrowband fibre gratings by phase mask scanning", Electronics Letters, 30 (16),1341-1342, (1994)

Kashyap, Fiber Bragg Gratings, Harcourt Brace \& Company, New York, 1999. 